\title{
RANDOM POLYTOPES IN SMOOTH CONVEX BODIES
}

\section{IMRE BÁRÁNY}

Abstract. Let $K \subset R^{d}$ be a convex body and choose points $x_{1}, x_{2}, \ldots, x_{n}$ randomly, independently, and uniformly from $K$. Then $K_{n}=\operatorname{conv}\left\{x_{1}, \ldots, x_{n}\right\}$ is a random polytope that approximates $K$ (as $n \rightarrow \infty$ ) with high probability. Answering a question of Rolf Schneider we determine, up to first order precision, the expectation of vol $K-\operatorname{vol} K_{n}$ when $K$ is a smooth convex body. Moreover, this result is extended to quermassintegrals (instead of volume).

\$1. Introduction and the theorems. Assume $K \subset R^{d}$ is a convex body (a convex compact set with nonempty interior) and let $x_{1}, \ldots, x_{n}$ be points chosen randomly, independently, and uniformly from $K$. Set $X_{n}=\left\{x_{1}, \ldots, x_{n}\right\}$ and call $K_{n}=\operatorname{conv} X_{n}$ a random polytope. In this paper we determine $E(K, n)$, the expectation of vol $\left(K \backslash K_{n}\right)$ for smooth convex bodies up to first order precision when $n$ tends to infinity.

The asymptotic behaviour of $E(K, n)$ has been known for different classes of convex bodies. Rényi and Sulanke [8 and 9] proved that for a polygon $P \subset R^{2}$

$$
E(P, n)=\frac{2}{3}(\# \text { vert } P)(\text { Area } P) \frac{\log n}{n}+O\left(\frac{1}{n}\right),
$$

and for smooth convex bodies $K \subset R^{2}$ (with positive curvature $\kappa$ )

$$
E(K, n)=\left(\frac{2}{3}\right)^{1 / 3} \Gamma\left(\frac{5}{3}\right) \int_{\partial K} \kappa^{1 / 3} d z\left(\frac{n}{\text { Area } K}\right)^{-2 / 3}+O\left(\frac{1}{n}\right) .
$$

Wieacker [13] determined the asymptotic behaviour of $E\left(B^{d}, n\right)$ where $B^{d}$ is the Euclidean unit ball of $\boldsymbol{R}^{d}$ :

$$
E\left(B^{d}, n\right)=c_{0}(d) n^{-2 /(d+1)}(1+o(1)),
$$

where $c_{0}(d)$ is an explicit constant. This can be improved using a recent result of Affentranger [1] to

$$
E\left(B^{d}, n\right)=c_{0}(d) n^{-2 /(d+1)}+O\left(n^{-4 /(d+1)}\right)
$$

(for $d \geqslant 3$ only, for $d=2$ one has (1.1)). This improvement is implicit in [1] and, in fact, if the computations there are carried out to second order precision then one obtains (1.2).

The asymptotic behaviour of $E(P, n)$ was determined by van Wel [14] and also by Affentranger and Wieacker [2] for simple polytopes $P \subset R^{d}$ and by Bárány and Buchta [4] for general ones.

In this paper we answer a question of Schneider and Wieacker [12, p. 69] which is repeated as a conjecture in Schneider [11, p. 222]. 
THEOREM 1. If $K \subset R^{d}$ is a $\mathscr{C}^{3}$ convex body with positive Gauss Kronecker curvature $\kappa$, then

$$
E(K, n)=c(d) \int_{\partial K} \kappa^{1 /(d+1)} d z\left(\frac{n}{\operatorname{vol} K}\right)^{-2 /(d+1)}+O\left(n^{-3 /(d+1)} \log ^{2} n\right),
$$

where $c(d)$ is a constant.

This has been known for $d=2$ and also for $d=3$ [13]. The idea behind the proof of the theorem is that $K$ is very close to an ellipsoid $E$ near $z \in \partial K$ and so $K_{n}$ is close to $E_{m}$, the random polytope in $E$ with $m$ points for some suitable $m$. The main difficulty in the proof is to show that $K_{n}$ near $z \in \partial K$ does not depend on the $x_{i}$ 's far from $z$. This means that the shape of $K_{n}$ near $z$ is "independent" of the shape of $K$ far from $z$-a fact that has been successfully applied to determine $E(P, n)$ where $P$ is a polytope (see [4]).

The same idea works when one wants to determine, up to first order precision, the expectation of the difference between the quermassintegrals of $K$ and $K_{n}$. Denote by $W_{i}^{(d)}(K)=W_{i}(K)$ the $i$-th quermassintegral of $K$ $(i=0,1, \ldots, d-1)$, see [5] or [10] for a definition. This includes and generalizes the cases of volume, surface area, and mean width that are constant multiples of $W_{0}, W_{1}$ and $W_{d-1}$. We introduce the notation

$$
E(K, i, n)=E\left(W_{i}(K)-W_{i}\left(K_{n}\right)\right) .
$$

Affentranger [1] determined $E\left(B^{d}, i, n\right)$ up to first order precision:

$$
E\left(B^{d}, i, n\right)=c_{0}(d, i) n^{-2 /(d+1)}(1+o(1)) .
$$

Here and in the next theorem, $o(1)$ could be replaced by $O\left(n^{-1 /(d+1)} \log ^{2} n\right)$, again.

THEOREM 2. If $K \subset R^{d}$ is a $C^{3}$ convex body with positive Gauss Kronecker curvature $\kappa$, then

$$
E(K, i, n)=c(d, i) \int_{\partial K} \kappa^{[i /(d-1)]+[1 /(d+1)]} d z\left(\frac{n}{\operatorname{vol} K}\right)^{-2 /(d+1)}(1+o(1)) .
$$

This has been known for $i=d-1$, i.e., for the mean width [12]. The case $i=0$ is Theorem 1 and the proof will be very similar so I will give only a sketch.

Let $f_{i}(P)$ denote the number of $i$-dimensional faces of the polytope $P \subset R^{d}$. It is clear (at least for the author) that there will be a similar theorem for the expectation of $f_{i}\left(K_{n}\right)$ and that this theorem will have the following form

$$
E f_{i}\left(K_{n}\right)=b(d, i) \int_{\partial K} \kappa^{1 /(d+1)} d z\left(\frac{n}{\operatorname{vol} K}\right)^{(d-1) /(d+1)}(1+o(1)),
$$

for some constant $b(d, i)$ provided $K$ satisfies the conditions of Theorem 1 (cf. [3]). (1.5) is known to be true for $i=d-1$ [13] and $i=d-2$ since $f_{d-2}=\frac{1}{2} d f_{d-1}$ for a simplicial $d$-polytope, and for $i=0$ it follows from Theorem 
1 and from an identity due to Efron [7] saying

$$
E f_{0}\left(K_{n}\right)=\frac{n}{\operatorname{vol} K} E(K, n-1) \text {. }
$$

As $K_{n}$ is a simplicial polytope with probability one the numbers $E f_{i}\left(K_{n}\right)$ satisfy the Dehn-Sommerville equations. This shows that conjecture (1.5) is true for $i=0,1, \ldots, d-1$ when $d=3,4,5$. When $d>5$ the conjecture is open even for $K=B^{d}$, the unit ball.

§2. Preliminaries. We will need several properties of smooth convex bodies. So assume $K \subset R^{d}$ is a $\mathscr{C}^{3}$ convex body with positive curvature $\kappa=\kappa(z)$ at every $z \in \partial K$. Then there is a constant $t_{0}>0$ such that $x \in K$, dist $(x, \partial K)=t \leqslant t_{0}$ implies $x$ can be written uniquely as

$$
x=z-t a
$$

where $z \in \partial K$ and $a$ is the outer unit normal to $K$ at $z$. Here $z, a, t$ all depend on $x$ but we will usually not denote this dependence. The constant $t_{0}$ depends on $K$ only. This will be true for all the constants $\Delta_{0}, b_{1}, b_{2}, \ldots, c_{1}, c_{2}, \ldots$, to come (unless stated otherwise).

Assume now that the principal radii of $K$ at $z \in \partial K$ are all equal, $R=R_{1}=R_{2}=\ldots=R_{d-1}=\kappa^{-1 /(d-1)}$. Let $H^{t}$ be the halfspace

$$
H^{t}=\left\{y \in R^{d}:(y-(z-a t)) . a \geqslant 0\right\},
$$

with the notation of (2.1). Also, write $B(y, r)$ for the ball with centre $y$ and radius $r$. Then, for $t \leqslant \Delta$

$$
H^{t} \cap B(z-(R-\Delta) a, R-\Delta) \subset H^{t} \cap K \subset H^{t} \cap B(z-(R+\Delta) a, R+\Delta),
$$

provided $\Delta \leqslant \Delta_{0}$ for some constant $\Delta_{0}>0$. Consequently

$$
b_{1}(R-\Delta)^{(d-1) / 2} t^{(d+1) / 2} \leqslant \operatorname{vol}\left(H^{t} \cap K\right) \leqslant b_{1}(R+\Delta)^{(d-1) / 2} t^{(d+1) / 2},
$$

where the constant $b_{1}$ depends only on $d$. Write $D=B(z-(R+\Delta) a, R+\Delta)$. We can estimate vol $\left(H^{\Delta} \cap K\right)$ with small error

$$
\left|\operatorname{vol}\left(H^{\Delta} \cap K\right)-\operatorname{vol}\left(H^{\Delta} \cap D\right)\right| \leqslant b_{2} \Delta^{(d+2) / 2} .
$$

Define now $u: K \rightarrow R$ by

$$
u(x)=\operatorname{vol}(K \cap(x-K)) .
$$

The region $K \cap(x-K)$ is centrally symmetric with respect to $x$. Moreover, if $x$ is close to $\partial K$ then $K \cap(x-K)$ is close to $\left(H^{t} \cap K\right) \cup\left(x-\left(H^{t} \cap K\right)\right)$ with $t$ and $H^{t}$ coming from (2.1) and (2.2). More precisely, for $x \in K$ and $t=\operatorname{dist}(x, \partial K)<t_{0}$

$$
b_{3} t^{(d+1) / 2} \leqslant u(x) \leqslant b_{4} t^{(d+1) / 2} .
$$

This follows from (2.4) if $K$ is "circular" around $z$ (i.e., $R_{1}=R_{2}=\ldots=R_{d-1}$ ). 
Otherwise (2.4) changes to

$$
b_{1}\left(\prod_{i=1}^{d-1}\left(R_{i}-\Delta\right)\right)^{1 / 2} t^{(d+1) / 2} \leqslant \operatorname{vol}\left(H^{t} \cap K\right) \leqslant b_{1}\left(\prod_{i=1}^{d-1}\left(R_{i}+\Delta\right)\right)^{1 / 2} t^{(d+1) / 2},
$$

and $\prod_{i=1}^{d-1} R_{i}=\kappa$ so (2.6) follows again.

A proof of the above facts can be found in [12] pages 71-72 of Schneider and Wieacker.

We will often use the following inequality (see [6] or [3] for a proof)

$$
\operatorname{Prob}\left(x \notin K_{n}\right) \leqslant 2 \sum_{i=0}^{d-1}\left(\begin{array}{l}
n \\
i
\end{array}\right)\left(\frac{u(x)}{2 \operatorname{vol} K}\right)^{i}\left(1-\frac{u(x)}{2 \operatorname{vol} K}\right)^{n-i} \text {. }
$$

Here Prob $\left(x \notin K_{n}\right)$ is meant with $x$ fixed and $K_{n}$, the random polytope in $K$ varying.

83. Proof of Theorem 1. We assume $d \geqslant 3$ (for $d=2$ see [8] or (1.1)) and also that vol $K=1$. The proof is split into several steps.

Step 1. The theorem is true for the ball $r B^{d}$.

Proof. If $K=r B^{d}$ then $\kappa=r^{-(d-1)}$ and (1.4) says

$$
\begin{aligned}
E\left(r B^{d}, n\right) & =c(d) \int_{\partial\left(r B^{d}\right)} \kappa^{1 /(d+1)} d z\left(\frac{n}{\omega_{d} r^{d}}\right)^{-2 /(d+1)}+O\left(n^{-3 /(d+1)} \log ^{2} n\right) \\
& =c(d) d \omega_{d}^{(d+3) /(d+1)} r^{d} n^{-2 /(d+1)}+O\left(n^{-3 /(d+1)} \log ^{2} n\right),
\end{aligned}
$$

which is correct according to (1.2).

Step 2. There is a constant $c_{1}$ such that with

$$
\begin{aligned}
t_{1} & =t_{1}(n)=c_{1}((\log n) / n)^{2 /(d+1)}, \\
E(K, n) & =\int_{x: t \leq t_{1}} \operatorname{Prob}\left(x \notin K_{n}\right) d x+O\left(n^{-1}\right),
\end{aligned}
$$

where the integration is taken over all $x \in K$ with $t=\operatorname{dist}(x, \partial K) \leqslant t_{1}$.

Proof. Clearly $E(K, n)=\int_{K} \operatorname{Prob}\left(x \notin K_{n}\right) d x$. According to (2.6) $t \geqslant t_{1}$ implies $u(x) \geqslant 3((\log n) / n)$ with $t_{1}=c_{1}((\log n) / n)^{2 /(d+1)}$. Then

$$
I=\int_{x: l \geq t_{1}} \operatorname{Prob}\left(x \notin K_{n}\right) d x \leqslant \int_{u(x) \geqslant 3(\log n) / n} \operatorname{Prob}\left(x \notin K_{n}\right) d x .
$$


Set $\lambda_{0}=\lceil 3 \log n\rceil$ and apply (2.7). Then, the same way as in [6],

$$
\begin{aligned}
I & \leqslant \int_{u(x) \geqslant 3(\log n) / n} 2 \sum_{i=0}^{d-1}\left(\begin{array}{c}
n \\
i
\end{array}\right)\left(\frac{u(x)}{2}\right)^{i}\left(1-\frac{u(x)}{2}\right)^{n-i} d x \\
& \leqslant \sum_{\lambda=\lambda_{0}}^{n} 2 \int_{(\lambda-1) / n \leqslant u(x) \leqslant \lambda / n} \sum_{0}^{d-1}\left(\begin{array}{l}
n \\
i
\end{array}\right)\left(\frac{u(x)}{2}\right)^{i}\left(1-\frac{u(x)}{2}\right)^{n-i} d x \\
& \leqslant \sum_{\lambda=\lambda_{0}}^{n} 2 \sum_{i=0}^{d-1}\left(\begin{array}{c}
n \\
i
\end{array}\right)\left(\frac{\lambda}{2 n}\right)^{i}\left(1-\frac{\lambda-1}{2 n}\right)^{n-i} \\
& \leqslant \sum_{\lambda=\lambda_{0}}^{n} 2 \sum_{i=0}^{d-1} \frac{\lambda^{i}}{2^{i} i !} \exp \left(-\frac{\lambda}{2}+\frac{d}{2}\right) \leqslant O\left(n^{-1}\right) .
\end{aligned}
$$

Let $x \in K$ with $x=z-t a$ as in (2.1). Assume $0 \leqslant t \leqslant t_{1}$. It is easy to see that

$$
d x=\left(1+O\left(\left(\frac{\log n}{n}\right)^{2 /(d+1)}\right)\right) d t d z
$$

Using this in (3.2) we get

$$
\begin{aligned}
\int_{x: t \leq t_{1}} & \operatorname{Prob}\left(x \notin K_{n}\right) d x \\
\quad & \int_{z \in \partial K} \int_{\substack{x=z-a t \\
0 \leqslant t \leqslant t_{1}}} \operatorname{Prob}\left(x \notin K_{n}\right) d t d z\left(1+O\left(\left(\frac{\log n}{n}\right)^{2 /(d+1)}\right)\right) .
\end{aligned}
$$

Near the point $z, K$ looks like an ellipsoid $E(z)$. If one applies an affine transformation $T=T(z)$ of determinant 1 that leaves every point of the line $z$-ta unchanged and the tangent plane to $K$ at $z$ invariant, then Prob $\left(x \notin K_{n}\right)=$ Prob $\left(x \notin(T K)_{n}\right)$ identically for every $x=z-t a, t \geqslant 0$. Choose such a $T$ that carries $E(z)$ to a ball $B(z)$. Clearly $B(z)=B(z-r a, r)$ where $r=\kappa^{-1 /(d-1)}$ with $\kappa=\kappa(z)$. From now on we assume that $T(z)$ has been applied at $z$. Set $m=\left\lfloor n \omega_{d} r^{d}\right\rfloor$. The basic idea of this proof is that $K_{n}$ is similar to $B(z)_{m}$ near the point $z$. More precisely our aim is to prove

$$
\begin{aligned}
& \left|\int_{x: t \leqslant t_{1}} \operatorname{Prob}\left(x \notin K_{n}\right) d x-\int_{x: t \leqslant t_{1}} \operatorname{Prob}\left(x \notin B(z)_{m}\right) d x\right| \\
& \quad=O\left(n^{-3 /(d+1)} \log ^{2} n\right) .
\end{aligned}
$$

Here in the second integral $x: t \leqslant t_{1}$ means $\left\{x \in B(z)\right.$ : dist $\left.(x, \partial B(z)) \leqslant t_{1}\right\}$. Notice that $t_{1}=c_{1}^{\prime}((\log m) / m)^{2 /(d+1)}$ for some constant $c_{1}^{\prime}$.

Step 3. (3.4) implies the theorem.

Proof. According to (3.1)

$$
E(B(z), m)=c(d) d \omega_{d}^{(d+3) /(d+1)} r^{d} m^{-2 /(d+1)}+O\left(m^{-3 /(d+1)} \log ^{2} m\right) .
$$


Step 2, applied to $K=B(z)$ and $n=m$ and (3.3) give

$$
E(B(z), m)=\left[\int_{\bar{z} \in \partial B(z)} \int_{\substack{x: t \leq t_{1} \\ x=\bar{x}-a t}} \operatorname{Prob}\left(x \notin B(z)_{m}\right) d t\right] d \bar{z}+O\left(m^{-1}\right) .
$$

The expression in the brackets does not depend on $\bar{z}$ so we get from the two representations of $E(B(z), m)$ that, with the particular choice $\bar{z}=z$,

$$
\begin{aligned}
& \int_{\substack{x: t \leq t_{1} \\
x=z-a t}} \operatorname{Prob}\left(x \notin B(z)_{m}\right) d t=c(d) \omega_{d}^{2 /(d+1)} r m^{-2 /(d+1)}+O\left(m^{-3 /(d+1)} \log ^{2} m\right) \\
& \quad=c(d) \kappa^{1 /(d+1)} n^{-2 /(d+1)}+O\left(n^{-3 /(d+1)} \log ^{2} n\right) .
\end{aligned}
$$

Then (3.2), (3.3), (3.4) and (3.5) prove that, indeed

$$
E(K, n)=c(d) \int_{\partial K} \kappa^{1 /(d+1)} d z n^{-2 /(d+1)}+O\left(n^{-3 /(d+1)} \log ^{2} n\right) .
$$

From now on I will drop $z$ from the notation if there is no ambiguity. So $B=B(z), a=a(z)$, etc. Set $\Delta=c_{2}((\log n) / n)^{2 /(d+1)}$ where $c_{2}$ will be fixed later and will be much larger than $c_{1}$. Recall notation (2.2),

$$
H^{t}=\left\{y \in R^{d}:(y-(z-a t)) . a \geqslant 0\right\} .
$$

Write $D=B(z-(r+\Delta) a, r+\Delta)$ and

$$
D^{\Delta}=D \cap H^{\Delta}, \quad B^{\Delta}=B \cap H^{\Delta}, \quad K^{\Delta}=K \cap H^{\Delta} .
$$

Then, by (2.3), (2.4) and (2.5) we get

$$
\begin{gathered}
D^{\Delta} \supset K^{\Delta}, \quad D^{\Delta} \supset B^{\Delta}, \\
\operatorname{vol}\left(D^{\Delta} \backslash K^{\Delta}\right) \leqslant b_{2} \Delta^{(d+2) / 2}, \\
\operatorname{vol}\left(D^{\Delta} \backslash B^{\dot{\Delta}}\right) \leqslant b_{2} \Delta^{(d+2) / 2}, \\
\operatorname{vol} D^{\Delta} \approx b_{1}(r+\Delta)^{(d-1) / 2} \Delta^{(d+1) / 2},
\end{gathered}
$$

provided $\Delta$ is small enough (i.e., $n$ is large enough).

Set $p=\left\lfloor n \omega_{d}(r+\Delta)^{d}\right\rfloor$. We mention at once that

$$
\left|\int_{x: t \leqslant t_{1}} \operatorname{Prob}\left(x \notin D_{p}\right) d x-\int_{x: t \leqslant t_{1}} \operatorname{Prob}\left(x \notin B_{m}\right) d x\right|=O\left(n^{-3(d+1)} \log ^{2} n\right) .
$$

This follows from (3.5) immediately. (Notice that $t_{1}=c_{1}^{\prime \prime}((\log p) / p)^{2 /(d+1)}$ and $t_{1}=c_{1}^{\prime}((\log m) / m)^{2 /(d+1)}$ with suitable constants $\left.c_{1}^{\prime}, c_{1}^{\prime \prime}.\right)$ This shows, in turn, that it is enough to prove (3.4) with $D$ and $p$ instead of $B$ and $m$, i.e.,

$$
\begin{aligned}
& \left|\int_{x: t \leqslant t_{1}} \operatorname{Prob}\left(x \notin K_{n}\right) d x-\int_{x: t \leq t_{1}} \operatorname{Prob}\left(x \notin D_{p}\right) d x\right| \\
& =O\left(n^{-3 /(d+1)} \log ^{2} n\right) .
\end{aligned}
$$


Next I show that $K_{n} \cap H^{\Delta}$ is essentially independent of the $x_{i}$ 's not lying in $H^{\Delta}$.

Step 4. If dist $(x, \partial K) \leqslant t_{1}$, then

$$
\mid \text { Prob }\left(x \notin \operatorname{conv}\left(X_{n} \cap K^{\Delta}\right)\right)-\operatorname{Prob}\left(x \notin \operatorname{conv} X_{n}\right) \mid=O\left(n^{-1}\right),
$$

where $X_{n}=\left\{x_{1}, \ldots, x_{n}\right\}$ is the random $n$-set from $K$.

We need the following lemma (see [4] for a similar statement with a similar, if more involved, application). We write ray $(x, y)=\{x+\tau y: \tau>0\}$.

LEMMA. If $x, x_{1}, \ldots, x_{n}$ are in general position and $x \in K^{\Delta} \cap$ conv $X_{n}$ but $x \notin \operatorname{conv}\left(X_{n} \cap K^{\Delta}\right)$, then there is an $x_{i} \in X_{n} \backslash K^{\Delta}$ with

$$
\operatorname{ray}\left(x, x_{i}\right) \cap \operatorname{conv}\left(X_{n} \backslash\left(K^{t} \cup\left\{x_{i}\right\}\right)\right)=\varnothing \text {. }
$$

Proof of the lemma. Identify $x$ with the origin for this proof. Then the conditions say that cone $X_{n}=R^{d}$ but cone $\left(X_{n} \cap K^{\Delta}\right) \neq R^{d}$ and cone $\left(X_{n} \backslash K^{\prime}\right) \neq R^{d}$. But cone $X_{n}=$ cone $\left(X_{n} \cap K^{\Delta}\right)+$ cone $\left(X_{n} \backslash K^{t}\right)$ so cone $\left(X_{n} \backslash K^{t}\right)$ must contain an extreme ray, defined by some $x_{i} \in X_{n} \backslash K^{t}$ which is not in cone $\left(X_{n} \cap K^{\Delta}\right)$. Then $x_{i} \notin X_{n} \cap K^{\Delta}$ as well and the ray does not meet conv $\left(X_{n} \backslash\left(K^{t} \cap\left\{x_{i}\right\}\right)\right)$ as required.

Proof of Step 4. Clearly

$$
\begin{aligned}
0 & \leqslant \operatorname{Prob}\left(x \notin \operatorname{conv}\left(X_{n} \cap K^{\Delta}\right)\right)-\operatorname{Prob}\left(x \notin \operatorname{conv} X_{n}\right) \\
& =\operatorname{Prob}\left(x \notin \operatorname{conv}\left(X_{n} \cap K^{\Delta}\right) \text { but } x \in \operatorname{conv} X_{n}\right) \\
& \leqslant \operatorname{Prob}\left(\exists x_{i} \in X_{n} \backslash K^{\Delta}: \operatorname{ray}\left(x, x_{i}\right) \cap \operatorname{conv}\left(X_{n} \backslash\left(K^{t} \cup\left\{x_{i}\right\}\right)\right)=\varnothing\right)
\end{aligned}
$$

(by the Lemma)

$$
\leqslant n \int_{y \in K \backslash K^{\Delta}} \operatorname{Prob}\left(\operatorname{ray}(x, y) \cap \operatorname{conv}\left(X_{n-1} \backslash K^{t}\right)=\varnothing\right) d y .
$$

Fix $t \in\left[0, t_{1}\right]$ and write $u_{t}$ for the $u$-function of the convex set $K \backslash K^{t}$. Let $y_{0} \in$ ray $(x, y)$ be the point maximizing $u_{t}$ on ray $(x, y)$. We claim that if $c_{2}$ is large enough then

$$
u_{t}\left(y_{0}\right) \geqslant 4 d(\log n) / n
$$

Indeed,

$$
u_{t}\left(y_{0}\right) \geqslant u\left(y_{0}\right)-2 u(x) .
$$

By (2.6) $u(x) \leqslant b_{4} t_{1}^{(d+1) / 2}$ and $u\left(y_{0}\right) \geqslant b_{3}\left[\operatorname{dist}\left(y_{0}, \partial K\right)\right]^{(d+1) / 2}$. As $K$ is very close to a ball near $z$, dist $\left(y_{0}, \partial K\right) \geqslant \Delta / 4$ follows quite easily. So

$$
\begin{aligned}
u_{t}\left(y_{0}\right) & \geqslant b_{3}\left(\frac{\Delta}{4}\right)^{(d+1) / 2}-2 b_{4} t_{1}^{(d+1) / 2}=\left(b_{3}\left(\frac{c_{2}}{4}\right)^{(d+1) / 2}-2 b_{4} c_{1}^{(d+1) / 2}\right) \frac{\log n}{n} \\
& \geqslant 4 d \frac{\log n}{n}
\end{aligned}
$$

as claimed if $c_{2}$ is large enough. Evidently $u_{t}\left(y_{0}\right) \leqslant 1$. 
Now with $\alpha=\operatorname{vol} K^{\prime}$ we get

$$
\begin{aligned}
& \operatorname{Prob}\left(\operatorname{ray}(x, y) \cap \operatorname{conv}\left(X_{n-1} \backslash K^{t}\right)=\varnothing\right) \\
& =\sum_{j=0}^{n-1} \operatorname{Prob}\left(\operatorname{ray}(x, y) \cap \operatorname{conv}\left(X_{n-1} \backslash K^{\prime}\right)=\varnothing|| X_{n-1} \cap K^{\prime} \mid=j\right) \\
& \times\left(\begin{array}{c}
n-1 \\
j
\end{array}\right) \alpha^{j}(1-\alpha)^{n-1-j} \\
& =\sum_{j=0}^{n-1} \operatorname{Prob}\left(\operatorname{ray}(x, y) \cap\left(K \backslash K^{t}\right)_{n-j-1}=\varnothing\right)\left(\begin{array}{c}
n-1 \\
j
\end{array}\right) \alpha^{j}(1-\alpha)^{n-1-j} \\
& \leqslant \sum_{j=0}^{n-1} \operatorname{Prob}\left(y_{0} \notin\left(K \backslash K^{t}\right)_{n-j-1}\right)\left(\begin{array}{c}
n-1 \\
j
\end{array}\right) \alpha^{j}(1-\alpha)^{n-1-j} \quad \text { (by (2.7)) } \\
& \leqslant \sum_{j=0}^{n-1} 2 \sum_{i=0}^{d-1}\left(\begin{array}{c}
n-j-1 \\
i
\end{array}\right)\left(\frac{u_{t}\left(y_{0}\right)}{2(1-\alpha)}\right)^{i}\left(1-\frac{u_{t}\left(y_{0}\right)}{2(1-\alpha)}\right)^{n-1-j-i} \\
& \times\left(\begin{array}{c}
n-1 \\
j
\end{array}\right) \alpha^{j}(1-\alpha)^{n-1-j} \\
& =2 \sum_{i=0}^{d-1}\left(\begin{array}{c}
n-1 \\
i
\end{array}\right)\left(\frac{u_{t}\left(y_{0}\right)}{2}\right)^{i}\left(1-\frac{u_{t}\left(y_{0}\right)}{2}\right)^{n-1-i} \\
& \leqslant 2 d n^{d-1}\left(1-\frac{4 d \log n}{2 n}\right)^{n-1}=O\left(n^{-2}\right) \text {. }
\end{aligned}
$$

Now we apply Step 4 with $K=D$ and $n=p$ to get

$$
\left|\operatorname{Prob}\left(x \notin \operatorname{conv}\left(Y_{p} \cap D^{\Delta}\right)\right)-\operatorname{Prob}\left(x \notin \operatorname{conv} Y_{p}\right)\right|=O\left(n^{-1}\right),
$$

where $Y_{p}=\left\{y_{1}, \ldots, y_{p}\right\}$ is a random $p$-set in $D$.

Write now $\beta=\operatorname{vol} K^{\Delta}$ and $\gamma=\operatorname{vol} D^{\Delta} /$ vol $D$. Clearly

$\operatorname{Prob}\left(x \notin \operatorname{conv}\left(X_{n} \cap K^{\Delta}\right)\right)$

$$
\begin{aligned}
& =\sum_{k=0}^{n} \operatorname{Prob}\left(x \notin \operatorname{conv}\left(X_{n} \cap K^{\Delta}\right)|| X_{n} \cap K^{\Delta} \mid=k\right)\left(\begin{array}{l}
n \\
k
\end{array}\right) \beta^{k}(1-\beta)^{n-k} \\
& =\sum_{k=0}^{n} \operatorname{Prob}\left(x \notin K_{k}^{\Delta}\right)\left(\begin{array}{l}
n \\
k
\end{array}\right) \beta^{k}(1-\beta)^{n-k}
\end{aligned}
$$

and analogously

$$
\operatorname{Prob}\left(x \notin \operatorname{conv}\left(Y_{p} \cap D^{\Delta}\right)\right)=\sum_{k=0}^{p} \operatorname{Prob}\left(x \notin D_{k}^{\Delta}\right)\left(\begin{array}{l}
p \\
k
\end{array}\right) \gamma^{k}(1-\gamma)^{p-k} .
$$

We know that $\beta \approx \gamma(r+\Delta)^{d} \omega_{d} \approx$ const $(\log n) / n$ and $p \approx n(r+\Delta)^{d} \omega_{d}$. The next two steps follow easily from the properties of the binomial distribution and the choice of $\gamma$ and $p$ (we omit the proofs). 
Step 5. There are numbers $k_{1}=\left\lfloor c_{3} \log n\right\rfloor$ and $k_{2}=\left\lceil c_{4} \log n\right\rceil$ with $k_{1}<k_{2}$ such that the contribution of the terms with $k<k_{1}$ and $k>k_{2}$ in both (3.10) and (3.11) is less than $O\left(n^{-1}\right)$.

Step 6. For $k=k_{1}, k_{1}+1, \ldots, k_{2}$

$$
\begin{aligned}
\left|\left(\begin{array}{l}
n \\
k
\end{array}\right) \beta^{k}(1-\beta)^{n-k}-\left(\begin{array}{l}
p \\
k
\end{array}\right) \gamma^{k}(1-\gamma)^{p-k}\right| \\
=\left(\begin{array}{l}
n \\
k
\end{array}\right) \beta^{k}(1-\beta)^{n-k}\left(1+O\left(n\left(\frac{\log n}{n}\right)^{(d+2) /(d+1)}\right)\right) .
\end{aligned}
$$

Step 7. For $t \in\left[0, t_{1}\right]$ and for $k=k_{1}, \ldots, k_{2}$

$$
\left|\operatorname{Prob}\left(x \notin K_{k}^{\Delta}\right)-\operatorname{Prob}\left(x \notin D_{k}^{\Delta}\right)\right|=O\left(\left(\frac{\log n}{n}\right)^{1 /(d+1)} \log n\right)
$$

Proof. Let $Z_{k}=\left\{z_{1}, \ldots, z_{k}\right\}$ denote the random $k$-set in $D^{\Delta}$. Set $\delta=\operatorname{vol}\left(D^{\Delta} \backslash K^{\Delta}\right) / \operatorname{vol} D^{\Delta}$. Then $\delta<b_{5} \Delta^{1 / 2}$ by (2.5) and (2.6). Define

$$
\begin{aligned}
& P_{1}=\left|\operatorname{Prob}\left(x \notin \operatorname{conv}\left(Z_{k} \cap K^{\Delta}\right)\right)-\operatorname{Prob}\left(x \notin K_{k}^{\Delta}\right)\right|, \\
& P_{2}=\left|\operatorname{Prob}\left(x \notin \operatorname{conv}\left(Z_{k} \cap K^{\Delta}\right)\right)-\operatorname{Prob}\left(x \notin D_{k}^{\Delta}\right)\right| .
\end{aligned}
$$

Clearly $\mid$ Prob $\left(x \notin K_{k}^{\Delta}\right)-\operatorname{Prob}\left(x \notin D_{k}^{\Delta}\right) \mid \leqslant P_{1}+P_{2}$. Moreover,

$$
\begin{aligned}
P_{1} \leqslant \sum_{j=0}^{k} \mid \operatorname{Prob}\left(x \notin \operatorname{conv}\left(Z_{k} \cap K^{\Delta}\right)|| Z_{k} \cap K^{\Delta} \mid=k-j\right) \\
-\operatorname{Prob}\left(x \notin K_{k}^{\Delta}\right) \mid\left(\begin{array}{l}
k \\
j
\end{array}\right) \delta^{j}(1-\delta)^{k-j} \\
=\sum_{j=0}^{k}\left|\operatorname{Prob}\left(x \notin K_{k-j}^{\Delta}\right)-\operatorname{Prob}\left(x \notin K_{k}^{\Delta}\right)\right|\left(\begin{array}{c}
k \\
j
\end{array}\right) \delta^{j}(1-\delta)^{k-j} \\
\leqslant \sum_{j=1}^{k}\left(\begin{array}{l}
k \\
j
\end{array}\right) \delta^{j}(1-\delta)^{k-j}<\sum_{j=1}^{k}(k \delta)^{j}<\frac{k \delta}{1-k \delta}=O\left(\left(\frac{\log n}{n}\right)^{1 /(d+1)} \log n\right) .
\end{aligned}
$$

Quite similarly,

$$
\begin{aligned}
P_{2} \leqslant \sum_{j=0}^{k} \mid \operatorname{Prob}\left(x \notin \operatorname{conv}\left(Z_{k} \cap K^{\Delta}\right)|| Z_{k} \cap K^{\Delta} \mid=k-j\right) \\
-\operatorname{Prob}\left(x \notin \operatorname{conv} Z_{k}|| Z_{k} \cap K^{\Delta} \mid=k-j\right) \mid\left(\begin{array}{c}
k \\
j
\end{array}\right) \delta^{j}(1-\delta)^{k-j} \\
=\sum_{j=1}^{k}|\operatorname{Prob}(\cdots)-\operatorname{Prob}(\cdots)|\left(\begin{array}{l}
k \\
j
\end{array}\right) \delta^{j}(1-\delta)^{k-j}=O\left(\left(\frac{\log n}{n}\right)^{1 /(d+1)} \log n\right) .
\end{aligned}
$$


Finally, we prove (3.7)

$$
\begin{aligned}
& \left|\operatorname{Prob}\left(x \notin K_{n}\right)-\operatorname{Prob}\left(x \notin D_{p}\right)\right| \\
& \quad \leqslant\left|\operatorname{Prob}\left(x \notin \operatorname{conv}\left(X_{n} \cap K^{\Delta}\right)\right)-\operatorname{Prob}\left(x \notin \operatorname{conv}\left(Y_{p} \cap D^{\Delta}\right)\right)\right|+O\left(n^{-1}\right) \\
& \quad \leqslant \sum_{k=k_{1}}^{k_{2}} \mid \operatorname{Prob}\left(x \notin K_{k}^{\Delta}\right)\left(\begin{array}{l}
n \\
k
\end{array}\right) \beta^{k}(1-\beta)^{n-k}-\operatorname{Prob}\left(x \notin D_{k}^{\Delta}\right) \\
& \quad \times\left(\begin{array}{l}
p \\
k
\end{array}\right) \gamma^{k}(1-\gamma)^{p-k} \mid+O\left(n^{-1}\right) \\
& \quad \leqslant \sum_{k=k_{1}}^{k_{2}}\left|\operatorname{Prob}\left(x \notin K_{k}^{\Delta}\right)-\operatorname{Prob}\left(x \notin D_{k}^{\Delta}\right)\right|\left(\begin{array}{l}
n \\
k
\end{array}\right) \beta^{k}(1-\beta)^{n-k} \quad+\left(n\left(\frac{\log n}{n}\right)^{(d+2) /(d+1)}\right) \\
& \leqslant \sum_{k=k_{1}}^{k_{2}} c_{5}\left(\frac{\log n}{n}\right)^{1 /(d+1)} \log n\left(\begin{array}{l}
n \\
k
\end{array}\right) \beta^{k}(1-\beta)^{n-k}+O\left(n\left(\frac{\log n}{n}\right)^{(d+2) /(d+1)}\right) \\
& =O\left(\left(\frac{\log n}{n}\right)^{1 /(d+1)} \log n\right) .
\end{aligned}
$$

Integrating this on $\left[0, t_{1}\right]$ with $t_{1}=c_{1}((\log n) / n)^{2 /(d+1)}$ we get (3.7).

§4. Sketch of the proof of Theorem 2. Recall first $[5,10]$ that

$$
W_{i}^{(d)}(K)=\int_{F \in G} \operatorname{vol}_{d-i}\left(\operatorname{proj}_{F}(K)\right) d \omega(F),
$$

where $G=G(d, d-i)$ is the Grassmannian of the $(d-i)$-dimensional subspaces of $R^{d}, \omega$ is the (unique) rotation-invariant measure on $G$ normalized suitably, and $\operatorname{proj}_{F}: R^{d} \rightarrow F$ denotes orthogonal projection onto $F \in G$. Then

$$
\begin{aligned}
E(K, i, n) & =E\left(W_{i}^{(d)}(K)-W_{i}^{(d)}\left(K_{n}\right)\right) \\
& =E \int_{G} \operatorname{vol}_{d-i}\left(\operatorname{proj}_{F}(K) \backslash \operatorname{proj}_{F}\left(K_{n}\right)\right) d \omega(F) \\
& =\int_{G} E \operatorname{vol}_{d-i}\left(\operatorname{proj}_{F}(K) \backslash \operatorname{proj}_{F}\left(K_{n}\right)\right) d \omega(F) \\
& =\int_{G} \int_{\bar{x} \in F} \operatorname{Prob}\left(\bar{x} \notin \operatorname{proj}_{F}\left(K_{n}\right)\right) d \bar{x} d \omega(F) .
\end{aligned}
$$

Now Step 1 follows as before using (1.3). Also, Step 2 goes the same way because Prob $\left(\bar{x} \notin \operatorname{proj}_{F}\left(K_{n}\right)\right)$ is very small when $\bar{x}$ is far from the boundary of $\operatorname{proj}_{F}(K)$. 
Write $t=\operatorname{dist}\left(\bar{x}, \partial \operatorname{proj}_{F}(K)\right), \bar{x}=\bar{z}-\bar{a} t$ with $\bar{z} \in \partial \operatorname{proj}_{F}(K)$ and $\bar{a}$ the outer unit normal to $\operatorname{proj}_{F}(K)$ at $\bar{z}$. Clearly $\bar{z}=\operatorname{proj}_{F} z$ for a unique $z \in \partial K$ where $a=\bar{a}$ is the outer normal to $K$ at $z$.

So we get from Step 2 and (4.1) with $t_{1} \leqslant c_{1}((\log n) / n)^{2 /(d+1)}$

$$
\begin{aligned}
E(K, i, n) & =\int_{G} \int_{\substack{\bar{x} \in F \\
t \leqslant t_{1}}} \operatorname{Prob}\left(\bar{x} \notin \operatorname{proj}_{F}\left(K_{n}\right)\right) d \bar{x} d \omega(F)+O\left(n^{-1}\right) \\
& =\int_{F \in G} \int_{\substack{z \in \partial K \\
a \in F}} \int_{\substack{\bar{x}=\bar{z}-t a \\
t \leqslant t_{1}}} \operatorname{Prob}\left(\bar{x} \notin \operatorname{proj}_{F}\left(K_{n}\right)\right) d t d z d \omega(F)+O\left(n^{-1}\right) \\
& =\int_{\substack { z \in \partial K \\
\begin{subarray}{c}{x=z-t a \\
i \in t_{1}{ z \in \partial K \\
\begin{subarray} { c } { x = z - t a \\
i \in t _ { 1 } } }\end{subarray}} \int_{\substack{F \in G \\
a \in F}} \operatorname{Prob}\left(\left(x+F^{\perp}\right) \cap K_{n}=\varnothing\right) d \omega(F) d t d z+O\left(n^{-1}\right),
\end{aligned}
$$

because $\bar{x} \notin \operatorname{proj}_{F}\left(K_{n}\right)$, if, and only if, $\left(x+F^{\perp}\right) \cap K_{n}=\varnothing$ where $x=z-t a$, $a \in F$. ( $F^{\perp}$ denotes the orthogonal complementary subspace of $F$.)

Apply now the same affine transformation $T$ as in the previous proof. Then, for $x=z-t a$ and $a \in F$,

$$
\operatorname{Prob}\left(\left(x+F^{\perp}\right) \cap K_{n}=\varnothing\right)=\operatorname{Prob}\left(\left(x+(T F)^{\perp}\right) \cap(T K)_{n}=\varnothing\right)
$$

identically in $x$. Our aim is to prove (with the same notation as earlier) that

$$
\begin{aligned}
& \int_{\substack { t \leq t_{1} \\
\begin{subarray}{c}{F \in G \\
a \in F{ t \leq t _ { 1 } \\
\begin{subarray} { c } { F \in G \\
a \in F } }\end{subarray}}\left(\operatorname{Prob}\left(\left(x+F^{\perp}\right) \cap K_{n}=\varnothing\right)-\operatorname{Prob}\left(\left(x+F^{\perp}\right) \cap B_{m}=\varnothing\right)\right) d \omega(F) d t \\
& \quad=o\left(n^{-2 /(d+1)}\right) .
\end{aligned}
$$

Now Step 3 says that (4.3) implies the theorem and the proof is analogous.

Set $A=x+F^{\perp}$. This is an $i$-dimensional affine subspace. For $y \in A \cap K$ dist $(y, \partial K) \leqslant t$ where $t$ comes from (2.1), i.e., from $x=z-t a$. Moreover,

$$
\max \{\operatorname{dist}(y, \partial K): y \in A \cap K\}=t .
$$

Letting $\Delta=c_{2}((\log n) / n)^{2 /(d+1)}$ again, Step 4 says that

$\left|\operatorname{Prob}\left(A \cap \operatorname{conv}\left(X_{n} \cap K^{\Delta}\right)=\varnothing\right)-\operatorname{Prob}\left(A \cap \operatorname{conv} X_{n}=\varnothing\right)\right|=o(1)$.

The proof of this follows the same lines. The auxiliary lemma we need here is

Lemma. If $A, x_{1}, x_{2}, \ldots, x_{n}$ are in general position and $A \cap K \subset K^{\Delta}$, $A \cap$ conv $X_{n} \neq \varnothing$ but $A \cap$ conv $\left(X_{n} \cap K^{\Delta}\right)=\varnothing$, then there exists an $x_{i} \subset X_{n} \backslash K^{\Delta}$ with

$$
\left(A+\operatorname{ray}\left(0, x_{i}-x\right)\right) \cap \operatorname{conv}\left(X_{n} \backslash\left(K^{t} \cup d\left\{x_{i}\right\}\right)\right)=\varnothing .
$$

The rest of the proof is the same. 


\section{References}

1. F. Affentranger. The convex hull of random points with spherically symmetric distribution. To appear in Rend. Sem. Mat. Torino.

2. F. Affentranger and J. A. Wieacker. On the convex hull of uniform random points in a simple $d$-polytope. Discrete and Comp. Geometry, 6 (1991), 191-205.

3. I. Bárány. Intrinsic volumes and $f$-vectors of random polytopes. Math. Ann., 285 (1989), 671-699.

4. I. Bárány and C. Buchta. On the convex hull of uniform random points in an arbitrary d-polytope. Auz. Öster. Akad. Wiss. Math.-Natur., 77 (1990), 25-27.

5. I. Bonnesen and W. Fenchel, Theorie der konvexen Körper (Springer, Berlin, 1934).

6. I. Bárány and D. G. Larman. Convex bodies, economic cap coverings, random polytopes. Mathematika, 35 (1988), 274-291.

7. B. Efron. The convex hull of a random set of points. Biometrika, 52 (1965), 331-343.

8. A. Rényi and $R$. Sulanke. Über die konvexe Hülle von $\mathbf{n}$ zufällig gewählten Punkten. Z. Wahrscheinlichkeitsth. verw. Geb., 2 (1963), 75-84.

9. A. Rényi and R. Sulanke. Über die konvexe Hülle von n zufällig gewählten Punkten II. Z. Wahrscheinlichkeitsth. verw. Geb., 3 (1964), 138-147.

10. L. A. Santaló. Integral Geometry and Geometric Probability (Addison-Wesley, Reading, MA, 1976).

11. R. Schneider. Random approximation of convex sets. J. Microscopy, 151 (1988), 211-227.

12. R. Schneider and J. A. Wieacker. Random polytopes in a convex body. $Z$. Walrscheinlichkeitsth. verw. Geb., 52 (1980), 69-73.

13. J. A. Wieacker. Einige Probleme der polyedrischen Approximation (Diplomarbeit, Freiburg i. Br., 1978).

14. B. F. van Wel. The convex hull of a uniform sample from the interior of a simple $d$-polytope. J. Appl. Prob., 26 (1989), 259-273.

Dr. I. Bárány,

The Mathematical Institute of the

Hungarian Academy of Sciences, 1364 Budapest P.O.B. 127, Hungary.
52A22: CONVEX AND DISCRETE GEOMETRY; General Convexity; Random convex sets and integral geometry.

Received on the 1 st of August, 1991. 